\title{
Low level of serum high-density lipoprotein cholesterol in gastric cancer correlates with cancer progression but not survival
}

\author{
Jian Guo Shen, Li Dan Jin, Min Jun Dong, Lin Bo Wang, Wen He Zhao, Jun Shen \\ Department of Surgical Oncology, Sir Run Run Shaw Hospital, Zhejiang University College of medicine, Hangzhou, China \\ Contributions: (I) Conception and design: JG Shen; (II) Administrative support: WH Zhao; (III) Provision of study materials or patients: JG Shen, LD \\ Jin, MJ Dong; (IV) Collection and assembly of data: None; (V) Data analysis and interpretation: None; (VI) Manuscript writing: All authors; (VII) \\ Final approval of manuscript: All authors. \\ Correspondence to: Jun Shen, MD. Department of Surgical Oncology, Sir Run Run Shaw Hospital, Zhejiang University College of medicine, \\ Hangzhou 310016, China. Email: shenjun0909@zju.edu.cn.
}

\begin{abstract}
Background: The clinical significance of lipid profile in gastric cancer remains unclear. The aim of the present study was to investigate the correlation between serum lipid profiles and patient clinical parameters as well as prognosis in gastric cancer.

Methods: The preoperative plasma lipid profile levels of 358 gastric cancer patients, who were operated in between 2001 and 2009, were retrospectively evaluated, and the correlation between these factors and patient clinical parameters as well as survival were analyzed.

Results: There was a significant association between serum high-density lipoprotein cholesterol level (HDL-C <54.2 mg/dL) and cancer progression, Logistic regression analysis revealed that lower level of serum HDL-C was an independent risk factor for deeper cancer invasion, nodal metastasis as well as late stage in patients with gastric cancer. However, no significant association was reported between other lipid markers [triglyceride (TG), total cholesterol (TC), low-density lipoprotein cholesterol (LDL-C), very lowdensity lipoprotein (VLDL) and apolipoprotein A (apoA)] and lymph node involvement as well as stage of disease. In univariate analysis and multivariate analyses regarding patient's survival, there was no significant association between the groups in terms of TG, TC, HDL-C, LDL-C, VLDL and apoA.
\end{abstract}

Conclusions: Low level of serum HDL-C in gastric cancer correlates with cancer progression but not patient's survival.

Keywords: Cancer aggression; gastric cancer; high-density lipoprotein cholesterol (HDL-C); survival

Submitted Feb 27, 2020. Accepted for publication Sep 04, 2020.

doi: $10.21037 /$ tcr-20-1220

View this article at: http://dx.doi.org/10.21037/tcr-20-1220

\section{Introduction}

Gastric cancer is now the fourth most common cancer worldwide, and it is the second most common cause of death from cancer. Almost two-thirds of the cases occur in developing countries and $42 \%$ in China alone $(1,2)$. Several pathological studies had demonstrated that tumor location, tumor size, depth of invasion and histological type were independent risk factors for lymph node metastasis and cancer progression (3-5). However, these data were all derived from pathological findings after surgery, and it's hard to estimate before surgery in this way. Factors that could be measured before surgery to evaluate the nodal status as well as tumor stage are of great help to determinate the extension of gastric surgery.

Various studies have suggested that increased dietary fat or cholesterol is correlated with increased risk for occurrence of certain cancers, such as gastrointestinal cancer, breast cancer and multiple myeloma (6-9). Previous reports have also shown the relationship between lipid profile and cancer progression. Notarnicola reported that elevated serum lipid levels might facilitate the development 
of distant metastasis in colorectal cancer patients (10). As for gastric cancer, the association between lipid profiles and lymph node status in gastric cancer was still controversial. Some studies reported that high levels of serum triglyceride (TG) or total cholesterol (TC) were associated with lymph node metastasis in gastric cancer $(11,12)$. However, others showed that low level of serum high-density lipoprotein cholesterol (HDL-C) and high TC/HDL-C ratio, but not TG and TC, were independent risk factors for lymph node metastasis in gastric cancer (13). Furthermore, the relationship between serum lipid profile levels and patient prognosis in gastric cancer was rarely studied. In this retrospective study, we investigated the preoperative serum lipid profiles, including TC, TG, HDL-C, LDL-C, VLDL and apoA and their correlations with cancer progression as well as patient's survival in 358 patients with gastric cancer, and tried to demonstrate the significance of lipid profiles in patient with gastric cancer. We present the following article in accordance with the STROBE reporting checklist (available at http://dx.doi.org/10.21037/tcr-20-1220).

\section{Methods}

\section{Patients}

A total of 368 patients diagnosed as gastric cancer pathologically admitted for surgery at the department of surgical oncology, Sir Run Run Shaw hospital, Zhejiang University College of Medicine, between May 2001 and June 2009, were reviewed. Among them, 10 patients without lipid profile records were excluded. Finally, 358 patients were retrospectively enrolled in this study.

Patient demographics and clinicopathologic characteristics, including tumor size, location, depth of invasion, tumor differentiation, node status and TNM stage were documented. TNM staging was classified based on the fifth edition of the International Union against Cancer and the American Joint Committee on Cancer (14). As for the tumor differentiation, well and moderately differentiated tubular adenocarcinoma, papillary adenocarcinoma and mucinous adenocarcinoma of the well-differentiated subtype were grouped as differentiated type; Poorly differentiated adenocarcinoma, signet ring cell carcinoma and mucinous adenocarcinoma of poorly differentiated subtype were grouped as undifferentiated type.

Neoadjuvant chemotherapy was usually performed in patients with locally advanced gastric cancer. Surgical managements for gastric cancer were usually performed according to the rules of the Japanese Research Society for Gastric Cancer. All specimens were histologically examined by means of H\&E staining.

Serum levels of TG, TC, HDL-C, LDL-C, VLDL, apoA were examined in early morning samples on the second day after admission and immediately measured using an auto-analyzer method.

The patients were followed up until death or until the date of last follow-up of Feb 28, 2015, and 123 patients $(34.3 \%)$ died during the follow up period.

\section{Statistical analysis}

All statistical calculations were carried out using SPSS 22.0 for Windows (Chicago, IL, USA). Receiver operating curve (ROC) analysis was performed to determine the optimal cutoff values for the lipid profile. Chi-square test was used for analyzing the relationship between the lipid profile and clinic-pathological parameters. Risk factors influencing the clinicopathological variables were determined by means of logistic regression analysis. Kaplan-Meier method was used to calculate the survival probabilities and log rank test was used to compare survival curves. Independent factors influencing the survival were determined by multivariate analysis using cox regression model. A P value of less than 0.05 was considered statistically significant.

The study was conducted in accordance with the Declaration of Helsinki (as revised in 2013). This study was approved by the ethics committee of Sir Run Run Shaw hospital (No. 20110226-90). Informed consent was obtained from all individual participants included in the study. The authors have no ethical conflicts to disclose.

\section{Results}

Among the total of 358 patients, three hundred patients $(83.8 \%)$ performed radical surgery, and others were treated palliatively. Patients general and clinicopathological characteristics are presented in Table 1 . The mean $\pm \mathrm{SD}$ serum TG, TC, HDL-C, LDL-C, VLDL and apoA levels in gastric cancer patients were $129.5 \pm 69.4,166.3 \pm 39.0$, $48.3 \pm 14.4,97.1 \pm 31.3,17.3 \pm 16.0$ and $14.7 \pm 14.2 \mathrm{mg} / \mathrm{dL}$ respectively. After performing receiver operating curve (ROC) analysis, the optimal cutoff value for TG was $110.3 \mathrm{mg} / \mathrm{dL}$ (AUC: $0.516,95 \% \mathrm{CI}: 0.452-0.581$ ), for TC was $182.5 \mathrm{mg} / \mathrm{dL}$ (AUC: $0.522,95 \%$ CI: $0.456-0.588$ ), for HDL-C was $54.2 \mathrm{mg} / \mathrm{dL}$ (AUC: 0.591, 95\% CI: $0.526-$ 0.657), for LDL-C was $117.5 \mathrm{mg} / \mathrm{dL}$ (AUC: $0.478,95 \%$ 
Table 1 Baseline patient characteristics

\begin{tabular}{lc}
\hline Characteristics & Values \\
\hline Age (years), mean \pm SD & $59.0 \pm 11.4$ \\
BMI, mean \pm SD & $21.4 \pm 3.1$ \\
Gender, n (\%) & \\
Male & $259(72.3)$ \\
Female & $99(27.7)$ \\
Tumor location, $\mathrm{n}(\%)$ & \\
Upper body & $62(17.3)$ \\
Middle body & $66(18.4)$ \\
Lower body & $218(60.9)$ \\
Whole stomach & $12(3.4)$ \\
Tumor size (cm), $\mathrm{n}(\%)$ & \\
$\leq 3$ & $82(23.2)$ \\
$>3$ & $55(15.5)$ \\
Tumor differentiation, $\mathrm{n}(\%)$ & $151(42.7)$ \\
Differentiated & $66(18.6)$ \\
Undifferentiated & $260(74.9)$ \\
pTNM, n (\%) & \\
III & $171(47.8)$ \\
\hline
\end{tabular}

CI: 0.410-0.546), for VLDL was $17.03 \mathrm{mg} / \mathrm{dL}$ (AUC: 0.513, 95\% CI: $0.447-0.578$ ) and for apoA was $9.71 \mathrm{mg} / \mathrm{dL}$ (AUC: 0.506, 95\% CI: 0.442-0.570).

We then analyzed the correlation between the cutoff values of lipid profile and the patient's clinical feature. Only the HDL-C, but not the TG, TC, HDL-C, LDL-C, VLDL and apoA, was associated with tumor invasion, lymph node metastasis and TNM stage, respectively (Table 2). Patients with HDL-C level less than $54.2 \mathrm{mg} / \mathrm{dL}$ showed deeper cancer invasion, more lymph node metastasis and aggressive stage as compared with those with HDL-C more than $54.2 \mathrm{mg} / \mathrm{dL}$ (Figure 1). Multivariate analysis using logistic regression model revealed that low level of serum HDL-C $(<54.20 \mathrm{mg} / \mathrm{dL})$ was one of the independent risk factors for serosal invasion (RR 1.808, $\mathrm{P}=0.037$ ), lymph node metastasis ( $R R$ 2.481, $\mathrm{P}=0.002$ ) and late stage ( $R R$ 2.544, $\mathrm{P}=0.010$ ), respectively (Table 3).
Univariate analysis was used to find out the prognostic factors in all candidate variables including clincopathological parameters and the aforementioned lipid profiles. Age, tumor size, tumor location, histological type, TNM stage were significantly associated with the patient survival. The mean survival time was 55.8 and 58.9 months in patients with high and low level of HDL-C, respectively, the difference was not statistically significant $(\mathrm{P}=0.810)$. Although we found a significant association of lipid profile including TG and VLDL with patient survival, multivariate analysis using Cox regression model showed that none of the lipid profiles we evaluated was an independent prognostic factor predicting the survival of gastric cancer (Table 4).

\section{Discussion}

Our study showed that the serum HDL-C level was associated with cancer progression in gastric cancer. However, the lipid profiles we evaluated in this study was not shown to be one of the independent factors for survival.

The biological role of HDL-C is less well understood than that of other lipoproteins. Previous studies have described a substantial decrease of serum HDL-C in gastrointestinal cancer patients, when compared with normal controls. Guo et al. also reported that low level of serum HDL-C was closely related to increased frequency of lymph node metastasis in gastric cancer (13). In his study, the cut-off level of HDL-C was $40 \mathrm{mg} / \mathrm{dL}$, which was referenced by the risk assessment criteria for chronic heart disease (15). Using receiver operating characteristic analysis to get the best sensitivity and specificity in the prediction of lymph node metastasis in gastric cancer, our results showed that, when divided by the cut-off level of HDL-C at $54.2 \mathrm{mg} / \mathrm{dL}$, patients with HDL-C level less than the cutoff value showed deeper cancer invasion, higher frequency of lymph node metastasis and later stage as compared with patients who had HDL-C level above $54.2 \mathrm{mg} / \mathrm{dL}$. These results were in consistence with previous studies, and may imply that serum HDL-C level may take a share in tumor progression. Although the exact reason is not clear, several researchers have provided evidence in support of this finding.

The major function of HDL-C is to remove excess cholesterol from peripheral cells, and to deliver them to the liver and steroidogenic cells for catabolism (16). As a result, a higher level of HDL-C leads to a lower level of cholesterol in peripheral cells and a lower one leads to a 
Table 2 Relationship between serum HDL-C and clinical characteristics in 358 patients with gastric cancer

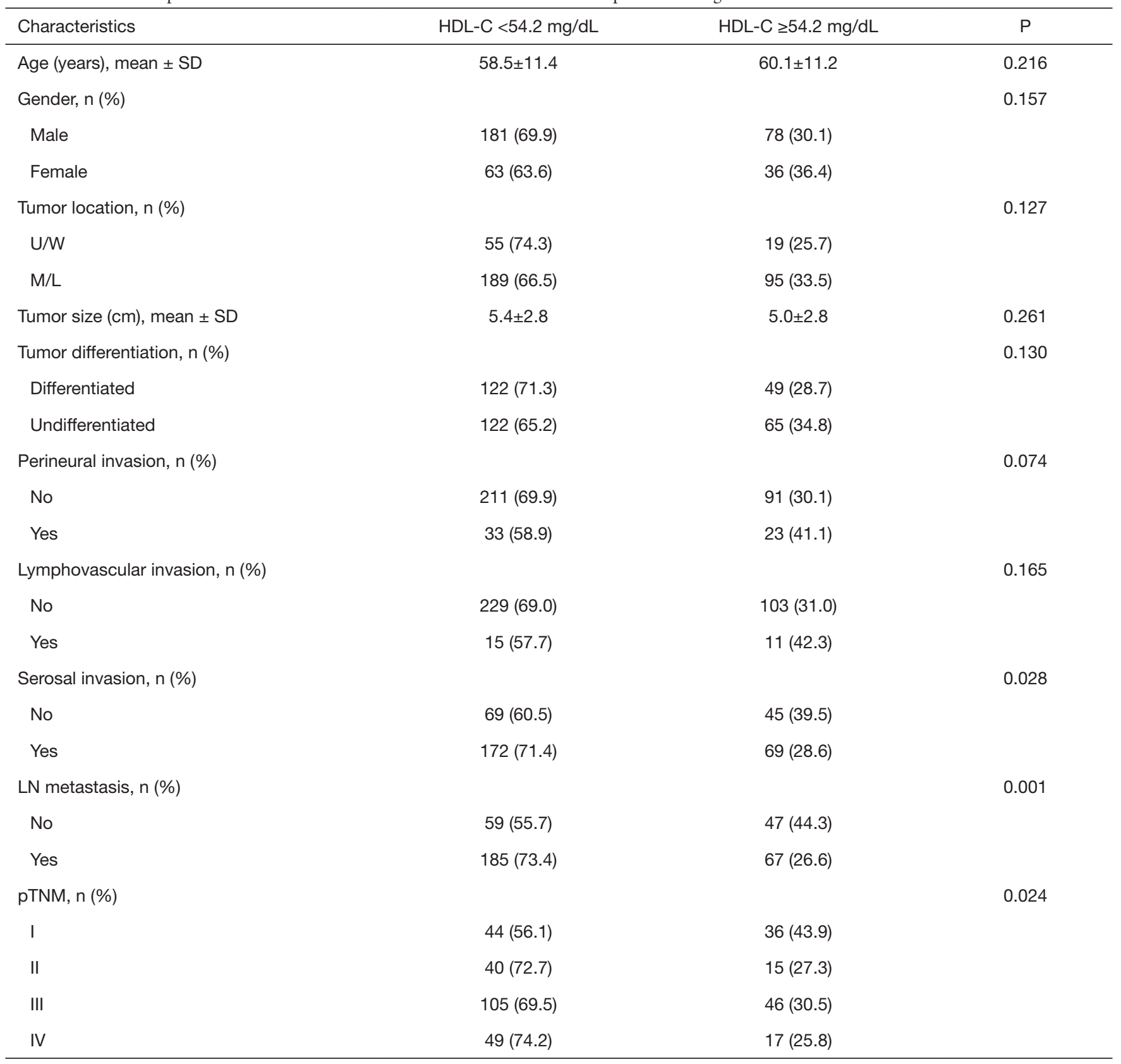

U/W, upper/whole body; M/L, middle/lower body.

higher one. Cholesterol is an indispensable ingredient for cell membrane. Many experimental studies have observed a positive association of tumor cell proliferation with cholesterol synthesis. Based on these data, we may infer that a lower level of cholesterol in peripheral cells, which may be caused by a higher level of HDL-C, restrain tumor development and lymphatic spread. On the other hand, studies showed that HDL-C level could be significantly influenced by interleukin- 1 and interleukin- 6 expression $(17,18)$. Increased expression of interleukin-1 and interleukin-6 might promote cancer progression in gastric cancer $(19,20)$. Based on this finding, we may infer that gastric cancer progression may increase the expression of interleukin-1 and interleukin-6, thus decreasing the serum 

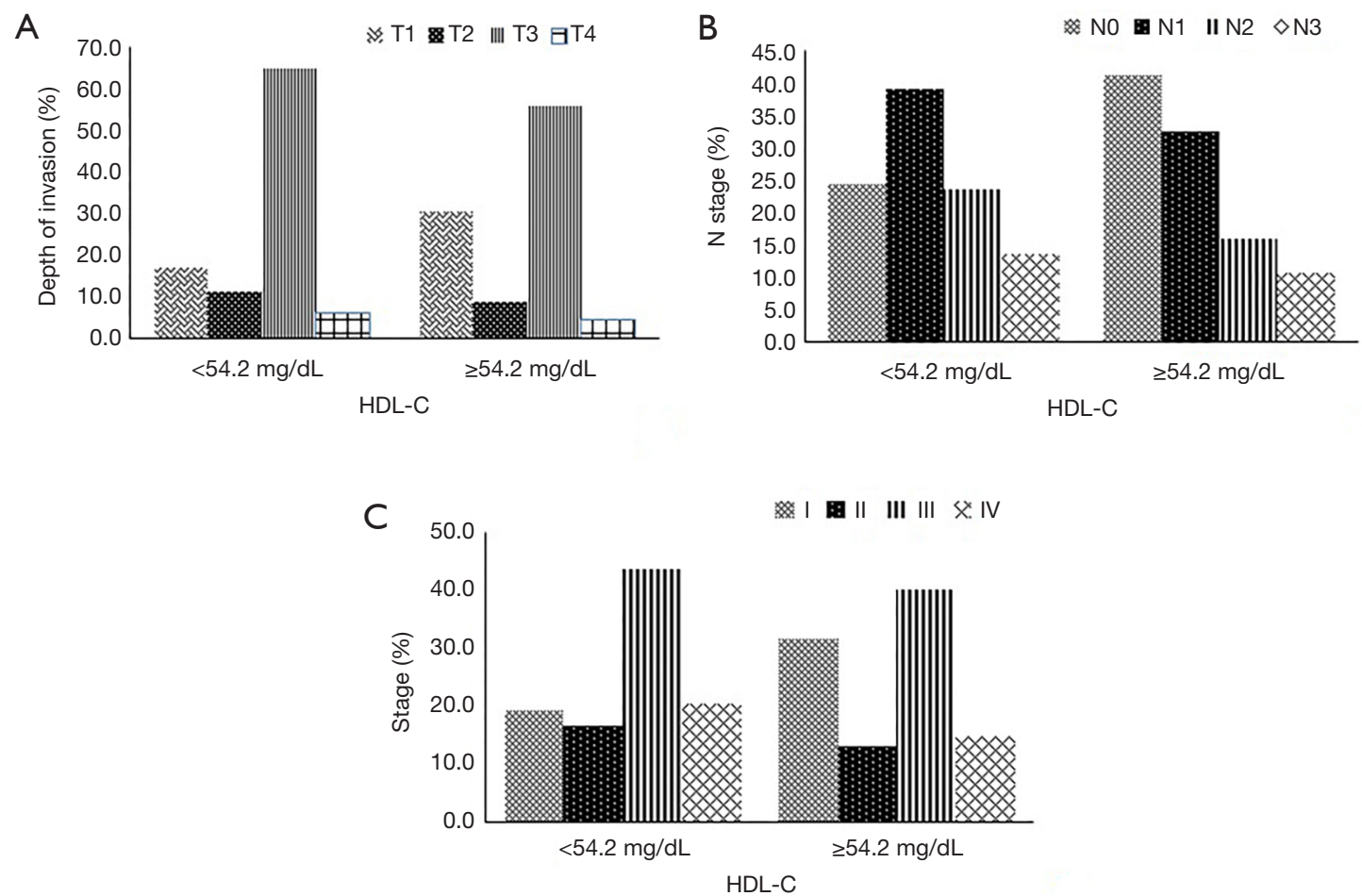

Figure 1 Distribution of depth of invasion (A), nodal involvement (B) and TNM stage (C) according to HDL-C values for 358 gastric cancers. Number of patients is given in parentheses.

Table 3 Logistic regression analyses of risk factors for serosal invasion, node-positive and late stage patients in gastric cancer, respectively

\begin{tabular}{|c|c|c|c|c|c|c|c|c|c|}
\hline Characteristics & \multicolumn{3}{|c|}{ Serosal invasion } & \multicolumn{3}{|c|}{ LN metastasis } & \multicolumn{3}{|c|}{ TNM stage } \\
\hline Age (<60 vs. $\geq 60$ years) & 0.458 & 0.266 to 0.789 & 0.005 & 0.925 & 0.526 to 1.627 & NS & 0.557 & 0.276 to 1.123 & NS \\
\hline Location (U/W vs. M/L) & 1.841 & 0.876 to 3.871 & NS & 0.620 & 0.308 to 1.247 & NS & 1.972 & 0.723 to 5.380 & NS \\
\hline Tumor size (>3 vs. $\leq 3 \mathrm{~cm}$ ) & 9.718 & 5.428 to 17.396 & 0.000 & 3.051 & 1.626 to 5.725 & 0.001 & 6.302 & 3.173 to 12.514 & 0.000 \\
\hline HDL-C ( $\geq 54.2$ vs. $<54.2 \mathrm{mg} / \mathrm{dL})$ & 1.808 & 1.038 to 3.151 & 0.037 & 2.481 & 1.398 to 4.404 & 0.002 & 2.544 & 1.254 to 5.161 & 0.010 \\
\hline Serosal invasion (no vs. yes) & - & - & - & 0.194 & 0.107 to 0.352 & 0.000 & 0.203 & 0.132 to 0.313 & 0.000 \\
\hline LN metastasis (no vs. yes) & - & - & - & - & - & - & 0.203 & 0.132 to 0.313 & 0.000 \\
\hline
\end{tabular}

U/W, upper/whole body; M/L, middle/lower body; RR, risk ratio; Cl, confidence interval; NS, no significant.

level of HDL-C. However, further studies are warranted to confirm this hypothesis.

In recent years, there has been increasing evidence that lipid profile correlates with the clinical outcome in patients with some cancers. Que et al. reported that decreased pre- operative serum HDL-C was associated with decreased DFS and OS in patients with soft tumor sarcoma following radical surgery (21). In prostate cancer, high HDL-C is regarded as a prognostic factor indicating a poor clinical outcome (22). As for gastric cancer, a study from Ma et al. 
Table 4 Univariate and multivariate analysis of gastric cancer with survival

\begin{tabular}{lccccc}
\hline \multirow{2}{*}{ Lipid profiles $(\mathrm{mg} / \mathrm{dL})$} & $\begin{array}{c}\text { Mean time } \\
\text { (months) }\end{array}$ & \multicolumn{2}{c}{ Univariate analysis } & & \multicolumn{2}{c}{ Multivariate analysis } \\
TG $(<110.3$ vs. $\geq 110.3)$ & 54.4 vs. 60.3 & $0.683(0.468-0.999)$ & 0.049 & $0.999(0.995-1.004)$ & 0.832 \\
TC $(<182.5$ vs. $\geq 182.5)$ & 56.7 vs. 60.3 & $0.722(0.472-1.105)$ & 0.134 & $0.994(0.972-1.016)$ & 0.590 \\
HDL-C $(<54.2$ vs. $\geq 54.2)$ & 58.9 vs. 55.8 & $0.952(0.636-1.424)$ & 0.810 & $1.006(0.978-1.034)$ & 0.696 \\
LDL-C $(<117.5$ vs. $\geq 117.5)$ & 57.3 vs. 60.7 & $0.786(0.490-1.261)$ & 0.318 & $1.002(0.978-1.026)$ & 0.879 \\
VLDL $(<17.03$ vs. $\geq 17.03)$ & 56.2 vs. 63.8 & $0.513(0.325-0.811)$ & 0.004 & $0.998(0.973-1.024)$ & 0.906 \\
apoA $(<9.71$ vs. $\geq 9.71)$ & 63.4 vs. 54.2 & $1.472(0.988-2.192)$ & 0.057 & $1.016(1.000-1.033)$ & 0.057 \\
\hline
\end{tabular}

showed that ApoB/ApoA1 could act as an independent prognostic marker in gastric cancer among all lipid molecules (23). Tamura et al. found a positive correlation between low serum HDL-C levels and prognosis for gastric cancer, patients in the low-HDL-C group (HDL-C $<40 \mathrm{mg} / \mathrm{dL}$ ) had a significantly higher rate of gastric cancer mortality compared with the normal-HDL-C group (HDL-C $\geq 40 \mathrm{mg} / \mathrm{dL}$ ) (24). However, Liu et al. reported that lipid markers including HDL-C, LDL-C, CHO, and TG do not present prognostic significance in gastric cancer (25). In the present study, TG and apoA were correlated with patient survival in univariate analysis. However, no lipid markers we mentioned above were found to be independent factors for prognosis in gastric cancer. The reasons for the controversial results of these studies may be explained as following: first, patients with different types of cancer might have different lipid profiles and thus the influence of lipid markers on cancer prognosis might be different. On the other hand, the factors analyzed for prognosis prediction in previous studies were variable, and this selection bias may also influence the outcome.

There were several limitations in this study. First, our results were based on a retrospective study with a relatively small number of patients. A large-scale analysis must be performed to further prove the findings. Furthermore, although some possible reasons have been used to explain the relationship between serum lipid profiles and cancer progression as well as prognosis, further experimental studies should be carried out to explore its intrinsic mechanism.

\section{Conclusions}

In conclusion, our study clearly indicates the potential risk marker of HDL-C level as an independent factor for cancer progression, although its role on patient prognosis is not fully identified.

\section{Acknowledgments}

Funding: None.

\section{Footnote}

Reporting Checklist: The authors present the study in accordance with the STROBE reporting checklist. Available at http://dx.doi.org/10.21037/tcr-20-1220

Data Sharing Statement: Available at http://dx.doi. org/10.21037/tcr-20-1220

Peer Review File: Available at http://dx.doi.org/10.21037/tcr20-1220

Conflicts of Interest: All authors have completed the ICMJE uniform disclosure form (available at http://dx.doi. org/10.21037/tcr-20-1220). All authors have no conflicts of interest to declare.

Ethical Statement: The authors are accountable for all aspects of the work in ensuring that questions related to the accuracy or integrity of any part of the work are appropriately investigated and resolved. The study was conducted in accordance with the Declaration of Helsinki (as revised in 2013). This study was approved by the ethics committee of Sir Run Run Shaw Hospital (No. 20110226-90). Informed consent was obtained from all individual participants included in the study. The authors have no 
ethical conflicts to disclose.

Open Access Statement: This is an Open Access article distributed in accordance with the Creative Commons Attribution-NonCommercial-NoDerivs 4.0 International License (CC BY-NC-ND 4.0), which permits the noncommercial replication and distribution of the article with the strict proviso that no changes or edits are made and the original work is properly cited (including links to both the formal publication through the relevant DOI and the license). See: https://creativecommons.org/licenses/by-nc-nd/4.0/.

\section{References}

1. Chen W, Zheng R, Baade PD. Cancer statistics in China, 2015. CA Cancer J Clin 2016;66:115-132.

2. Thrift AP, El-Serag HB. Burden of Gastric Cancer. Clin Gastroenterol Hepatol 2020;18:534-42.

3. Haruta H, Hosoya Y, Sakuma K, et al. Clinicopathological study of lymph-node metastasis in 1,389 patients with early gastric cancer: Assessment of indications for endoscopic resection. J Dig Dis 2008;9:213-8.

4. Wu L, Liang Y, Zhang C, et al. Prognostic significance of lymphovascular infiltration in overall survival of gastric cancer patients after surgery with curative intent. Chin J Cancer Res 2019;31:785-96.

5. Chen QY, Hong ZL, Zhong Q, et al. Nomograms for pre- and postoperative prediction of long-term survival among proximal gastric cancer patients: A large-scale, single-center retrospective study. World J Clin Cases 2019;7:3419-35.

6. Fichtali K, Bititi A, Elghanmi A, et al. Serum Lipidomic Profiling in Breast Cancer to Identify Screening, Diagnostic, and Prognostic Biomarkers. Biores Open Access 2020;9:1-6.

7. Fiorenza AM, Branchi A, Sommariva D. Serum lipoprotein profile in patients with cancer. A comparison with noncancer subjects. Int J Clin Lab Res 2000;30:141-5.

8. Liang L, Li J, Fu H, et al. Identification of High Serum Apolipoprotein A1 as a Favorable Prognostic Indicator in Patients with Multiple Myeloma. J Cancer 2019;10:4852-9.

9. Tomiki Y, Suda S, Tanaka M, et al. Reduced low-densitylipoprotein cholesterol causing low serum cholesterol levels in gastrointestinal cancer: A case control study. J Exp Clin Cancer Res 2004;23:233-40.

10. Notarnicola M, Altomare DF, Correale M, et al. Serum lipid profile in colorectal cancer patients with and without synchronous distant metastases. Oncology 2005;68:371-4.

11. Kitayama J, Hatano K, Kaisaki S, et al. Hyperlipidaemia is positively correlated with lymph node metastasis in men with early gastric cancer. Br J Surg 2004;91:191-8.

12. Kitayama J, Tabuchi M, Tsurita G, et al. Adiposity and gastrointestinal malignancy. Digestion 2009;79 Suppl 1:26-32.

13. Guo E, Chen L, Xie Q, et al. Serum HDL-C as a potential biomarker for nodal stages in gastric cancer. Ann Surg Oncol 2007;14:2528-34.

14. Sobin LH, Wittekind C. International union against cancer (UICC). Tnm classification of malignant tumors. 5th ed. New York: Wiley, 1997:59-62.

15. National Cholesterol Education Program (NCEP) Expert Panel on Detection, Evaluation, and Treatment of High Blood Cholesterol in Adults (Adult Treatment Panel III). Third report of the national cholesterol education program (ncep) expert panel on detection, evaluation, and treatment of high blood cholesterol in adults (adult treatment panel iii) final report. Circulation 2002;106:3143-421.

16. Ganjali S, Ricciuti B, Pirro M, et al. High-Density Lipoprotein Components and Functionality in Cancer: State-of-the-Art. Trends Endocrinol Metab 2019;30:12-24.

17. Hardardóttir I, Moser AH, Memon R, et al. Effects of TNF, IL-1, and the combination of both cytokines on cholesterol metabolism in Syrian hamsters. Lymphokine Cytokine Res 1994;13:161-6.

18. Yang W, Shen Z, Wen S, et al. Mechanisms of multiple neurotransmitters in the effects of Lycopene on brain injury induced by Hyperlipidemia. Lipids Health Dis 2018;17:13.

19. Ikeguchi $M$, Hatada T, Yamamoto $M$, et al. Serum interleukin-6 and -10 levels in patients with gastric cancer. Gastric Cancer 2009;12:95-100.

20. Uefuji K, Ichikura T, Mochizuki H. Increased expression of interleukin- 1 alpha and cyclooxygenase- 2 in human gastric cancer: A possible role in tumor progression. Anticancer Res 2005;25:3225-30.

21. Que Y, Jiang F, Liu L, et al. Clinical significance of preoperative serum high density lipoprotein cholesterol levels in soft tissue sarcoma. Medicine (Baltimore) 2015;94:e844.

22. Kotani K, Sekine Y, Ishikawa S, et al. High-density lipoprotein and prostate cancer: an overview. J Epidemiol 2013;23:313-9.

23. Ma MZ, Yuan SQ, Chen YM, et al. Preoperative apolipoprotein $\mathrm{B}$ / apolipoprotein $\mathrm{A} 1$ ratio: a novel 
prognostic factor for gastric cancer. Onco Targets Ther 2018;11:2169-76.

24. Tamura T, Inagawa $S$, Hisakura K, et al. Evaluation of serum high-density lipoprotein cholesterol levels as a prognostic factor in gastric cancer patients. J Gastroenterol
Hepatol 2012;27:1635-40.

25. Liu BZ, Tao L, Chen YZ, et al. Preoperative Body Mass Index, Blood Albumin and Triglycerides Predict Survival for Patients with Gastric Cancer. PLoS One 2016;11:e0157401.

Cite this article as: Shen JG, Jin LD, Dong MJ, Wang LB, Zhao WH, Shen J. Low level of serum high-density lipoprotein cholesterol in gastric cancer correlates with cancer progression but not survival. Transl Cancer Res 2020;9(10):6206-6213. doi: $10.21037 /$ tcr-20-1220 